\title{
Trajectory Research about the Rolling-Pin Belt Transmission
}

\author{
Huiyong Zhao* and Qingyong Zhang
}

Hubei University of Automotive Technology, Shiyan, 442002, China

\begin{abstract}
A new belt transmission mechanism, having the function of no-slip driving, is described in the paper. It is composed of pulleys with carved rails and belt with rolled pins spaced out on both sides. Firstly, the trajectory curve is deduced along which pins embedded in the pulley. And then, parametric relationships are established, which are about center offsets of two pulleys, working radius of each pulley, the number of meshing curves on every pulley and the number of pins on the belt. All of those provide a theoretical basis for the design of this type of no-slip belt transmission mechanisms.
\end{abstract}

Keywords: Rolling pin, separate curve, binding curve, mechanism.

\section{INTRODUCTION}

With the priorities of high efficiency and compact layout, mechanical belt transmission plays a very important role in mechanical engineering, and is widely used in automobile engines, gearboxes and other power transmissions [1-3]. As the traditional belt drive belongs to the friction drives, there are many well-known problems such as low transmission power, high slipping rate during transmission, easily failing and so on. Synchronizer gear-belt is a generally used alternative way to solve it [4-7]. However, the strength of the synchronizer gear-belt is limited to a very low level. Therefore, this paper proposes a new flexural transmission mecha- particularly defined, coordinate system $S_{l}=\left[\mathrm{O}_{1} ; x, y\right]$ is the left pulley coordinate system, $S_{r}=\left[\mathrm{O}_{r} ; x, y\right]$ is that of the right one [8]. Coordinate system $S=[\mathrm{O} ; x, y]$ is a fixed one whose origin superimposes with the left pulley's center, and $S^{\prime}=\left[\mathrm{O}^{\prime} ; x, y\right]$ is another fixed coordinate system whose origin locates at the right pulley's center. $\alpha_{l}$ and $\alpha_{r}$ are the angles which describe $x$-axis to the lines connecting the initial integration point with the origin of pulley. So the following relation formulas can be obtained immediately.

$$
\alpha=\arcsin \frac{\left(r_{r}-r_{l}\right)}{a}
$$
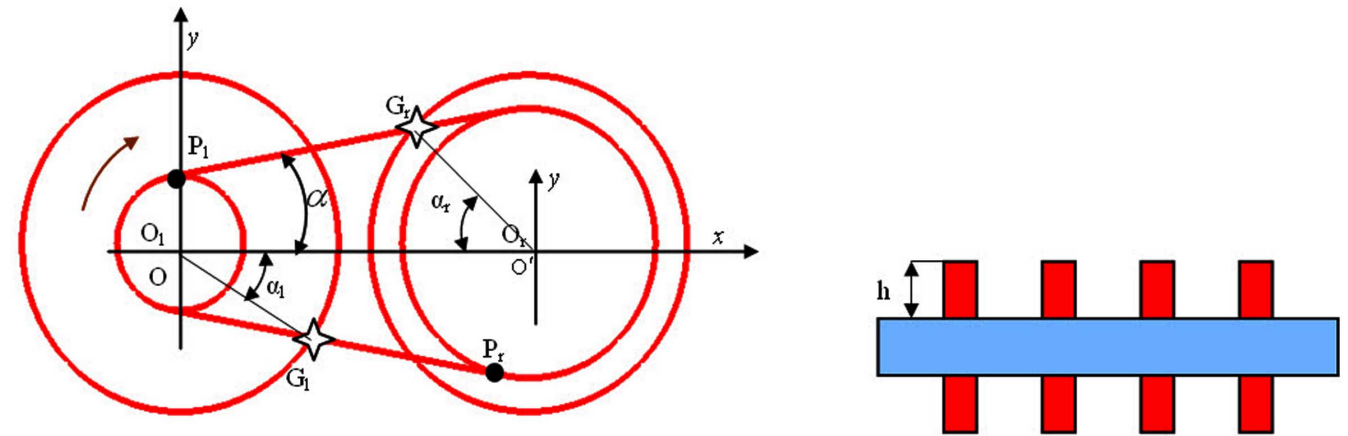

Fig. (1). Architecture diagram of belt transmission.

nism to improve the efficiency and transmitted power, and also to avoid elastic slip by embedding the rolling pins in the belt.

\section{INTRODUCTION TO THE NEW BELT DRIVE STRUCTURE}

As shown in Fig. (1), the belt is assembled with two pulleys and the belt is embedded with evenly distributed rolling pins. The center coordinate system on each pulley is

*Address correspondence to this author at the Department of Automotive Engineering, Hubei University of Automotive Technology, Shiyan, 442002, China; Tel: +86-0719- 8207271(office), +86-13636159823 (Mobile); Fax: +86-0719-8239594; E-mails: zhy9823@yahoo.com.cn,38580753@qq.com where, $\alpha$ denotes the angle between belt and $x$-axis in coordinate system $\mathrm{S}, r_{l}$ and $r_{r}$ represents working radius of left pulley and the right one respectively, $a$ indicates distance between the center of left pulley and that of the right pulley $[9,10]$.

Point $\mathrm{P}_{1}\left(x_{0 p l}, y_{0 p 1}\right)$ is the initial separation point of the left pulley, $\mathrm{P}_{\mathrm{r}}\left(x_{0 p r}, y_{0 p r}\right)$ is the similar point of the right one. From the simple geometric relationship, coordinates of point $\mathrm{P}_{1}$ and $\mathrm{P}_{\mathrm{r}}$ are described in the following formulas.

$$
\begin{aligned}
& x_{0 p l}=-r_{l} \sin (\alpha) \\
& y_{0 p l}=r_{l} \cos (\alpha) \\
& x_{0 p r}=-r_{r} \sin (\alpha)
\end{aligned}
$$


$y_{0 p r}=-r_{r} \cos (\alpha)$

where, $x_{0 p l}$ and $y_{0 p l}$ is $x$-coordinate and $y$-coordinate of point $\mathrm{P}_{1}$ in coordinate system $\mathrm{S}, x_{0 p}$ and $y_{0 p \mathrm{r}}$ are $x$-coordinate and $y$ coordinate of point $\mathrm{P}_{\mathrm{r}}$ in coordinate system $\mathrm{S}$, respectively.

In order to find the relationship between $\alpha_{l}$ and $\alpha$, geometric relation between point $\mathrm{G}_{\mathrm{r}}\left(x_{0 g r}, y_{0 g r}\right)$ and coordinate system $\mathrm{S}$ is shown in Fig. (2) where $\alpha_{l}$ is represented by formula (6). Similarly, relationship between $\alpha_{r}$ and $\alpha$ is specified with formula (7).

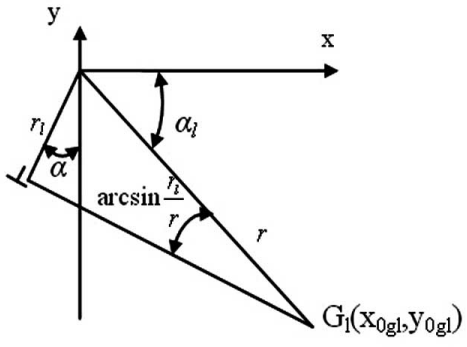

(a) Left pulley

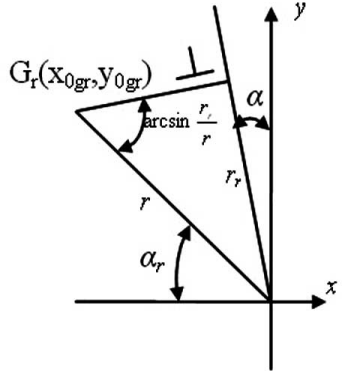

(b)Right pulley
Fig. (2). Geometric relation diagram.

$\alpha_{l}=\arcsin \frac{r_{l}}{r}+\alpha$

$\alpha_{r}=\arcsin \frac{r_{r}}{r}-\alpha$

where, $r$ is the radius of each pulley.

$\mathrm{G}_{1}\left(x_{0 g l}, y_{0 g l}\right)$ is the initial integration point of the left pulley, $\mathrm{G}_{\mathrm{r}}\left(x_{0 \mathrm{gr}}, y_{0 \mathrm{gr}}\right)$ is the corresponding point of the right one. Immediately, coordinates of point $G_{1}$ and point $G_{r}$ can be obtained in the following formulas.

$x_{0 g l}=r \cos \left(\alpha_{l}\right)$

$y_{0 g l}=-r \sin \left(\alpha_{l}\right)$

$x_{0 g r}=-r \cos \left(\alpha_{r}\right)$

$y_{0 g r}=r \sin \left(\alpha_{r}\right)$
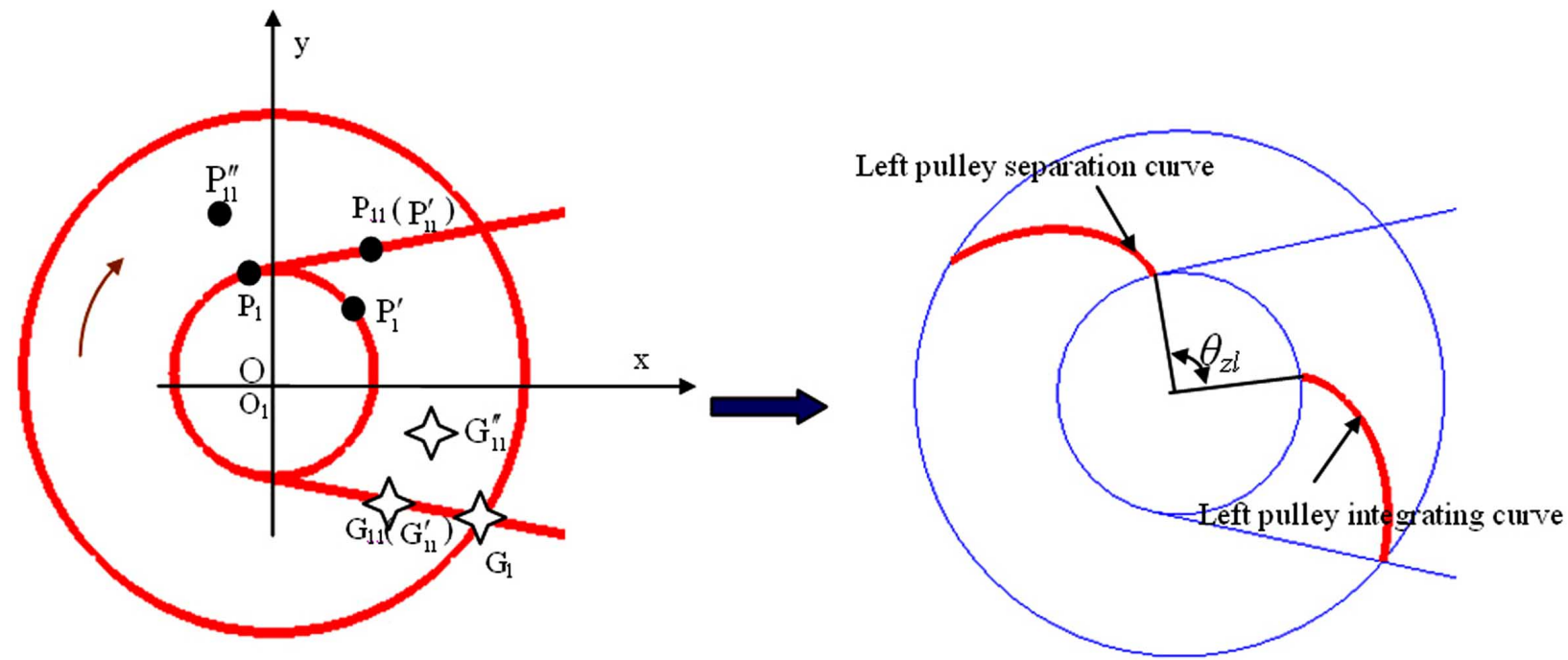

where, $x_{0 g l}$ and $y_{0 g l}$ are the corresponding $x$-coordinate and $y$ coordinate of point $\mathrm{G}_{1}$ in coordinate system $\mathrm{S}, x_{0 \mathrm{gr}}$ and $y_{0 \mathrm{gr}}$ are the corresponding $x$-coordinate and $y$-coordinate of point $\mathrm{G}_{\mathrm{r}}$ in coordinate system $\mathrm{S}$.

\section{LEFT PULLEY CURVE EQUATION}

\subsection{Equation of the Left Pulley Separation Curve}

In Fig. (3), $\omega_{l}$ is the angular velocity of the left pulley, point $\mathrm{P}_{11}\left(x_{l p l}, y_{l p l}\right)$ and point $\mathrm{P}_{1}^{\prime}$ are new places of point $\mathrm{P}_{1}$ on belt and pulley in the fix coordinates system $\mathrm{S}$ at time $t$, and $\theta_{l}$ represents the value of $\angle \mathrm{P}_{1} \mathrm{O}_{1} \mathrm{P}_{1}^{\prime}$. While the rolling pin runs from the separation point $\mathrm{P}_{1}$ to the point $\mathrm{P}_{11}$, point $\mathrm{P}_{11}^{\prime \prime}$ on the pulley rotates to the point $\mathrm{P}_{11}^{\prime}$ which superimposes with point $\mathrm{P}_{11}$ in coordinate system $\mathrm{S}$, and point $\mathrm{P}_{1}$ on the belt revolves to the point $\mathrm{P}_{1}^{\prime}$ in coordinate system $\mathrm{S}$. So the relations about $\omega_{l}$ and $\theta_{l}$ are represented in the following formulas.

$\omega_{l}=\frac{\sqrt{\left(r^{2}-r_{l}^{2}\right)}}{r_{l} t_{0 l}}$

$\theta_{l}=\omega_{l} t \frac{180}{\pi}$

where, $t_{0 l}$ is the total separation time, $t$ denotes running time during separation process.

From kinematics relationship, the speed of rolling pin is $r_{l} \omega_{l}$. According to the geometric relationship, coordinate calculating relationships of point $\mathrm{P}_{11}$ are described as:

$$
\begin{aligned}
& x_{1 p l}=x_{0 p l}+r_{l} \omega_{l} t \cos (\alpha) \\
& y_{1 p l}=y_{0 p l}+r_{l} \omega_{l} t \sin (\alpha)
\end{aligned}
$$

where, $x_{1 g l}$ and $y_{1 g l}$ are the corresponding $x$-coordinate and $y$ coordinate of point $\mathrm{P}_{11}$ in coordinate system $\mathrm{S}$.

At this time, $\angle \mathrm{P}_{1} \mathrm{O}_{1} \mathrm{P}_{1}^{\prime}$ is the angle that coordinate system $\mathrm{S}_{l}$ rotates relative to coordinate system $\mathrm{S}$, which is equal to the value of $\angle \mathrm{P}_{11}^{\prime \prime} O_{1} \mathrm{P}_{11}^{\prime}$. So it is deduced that the coordinate of $\mathrm{P}_{11}^{\prime \prime}$ is the position that $\mathrm{P}_{11}$ rotates $\theta_{l}$ counterclockwise around the coordinate origin.

Fig. (3). Points relation diagram of left pullet.

Fig. (3). Points relation diagram of left pullet. 
Defining the coordinate of point $\mathrm{P}_{11}^{\prime \prime}$ as $\left(x_{p l}, y_{p l}\right)$ in coordinate system $\mathrm{S}_{l}$ and coordinate transformation matrix as $\mathrm{A}_{l}$, the coordinate $\left(x_{p l}, y_{p l}\right)$ is described as:

$$
\left(\begin{array}{ll}
x_{p l} & y_{p l}
\end{array}\right)^{\mathbf{T}}=\mathbf{A}_{l}\left(\begin{array}{ll}
x_{1 p l} & y_{p l 1}
\end{array}\right)^{\mathbf{T}}
$$

where, $\mathrm{A}_{l}$ is the coordinate transformation matrix from coordinate system $\mathrm{S}$ to $\mathrm{S}_{l}[11,12]$, the matrix is described as,

$$
A_{l}=\left[\begin{array}{cc}
\cos \theta_{l} & -\sin \theta_{l} \\
\sin \theta_{l} & \cos \theta_{l}
\end{array}\right]
$$

\subsection{Equation the Left Pulley Integrating Curve}

As described in the Fig. (3), point $\mathrm{G}_{1}\left(x_{0 g l}, y_{0 g l}\right)$ is the initial integration point between the left pulley and belt, $\mathrm{G}_{11}\left(x_{1 g l}, y_{1 g l}\right)$ is some point before the end of integration. Because point $G_{1}$ is starting integration while point $P_{1}$ starts separation, rotating speed and the time are equal that two points spend on respective integrating and separating, which are named as $t_{0 l}$ and $\omega_{l}$ above. According to the geometric relationship, the coordinate of Point $\mathrm{G}_{11}$ is described as:

$x_{1 g l}=x_{0 g l}-r_{l} \omega_{l} t \cos (\alpha)$

$y_{1 g l}=y_{0 g l}+r_{l} \omega_{l} t \sin (\alpha)$

where, $x_{1 g l}$ and $y_{1 g l}$ are corresponding $x$-coordinate and $y$ coordinate of point $\mathrm{G}_{11}$ in coordinate system $\mathrm{S}$.

While rolling pin runs from the initial integration point $\mathrm{G}_{1}$ to the point $G_{11}$, point $G_{11}^{\prime \prime}$ on the pulley rotates to the point $\mathrm{G}_{11}^{\prime}$ which superimposes with point $\mathrm{G}_{11}$ in the fixed coordinate system $\mathrm{S}$, and the pulley coordinate system $\mathrm{S}_{l}$ also rotates angle $\theta_{l}$ relative to the original fixed coordinate system $\mathrm{S}$. So it is deduced that the coordinate of $\mathrm{G}_{11}^{\prime \prime}$ is the position that $\mathrm{G}_{11}$ rotates $\theta_{l}$ counterclockwise around the point $\mathrm{O}_{1}$. According to the geometric relationship, the coordinate of point $\mathrm{G}_{11}^{\prime \prime}$ is described as:

$\left(\begin{array}{ll}x_{g l} & y_{g l}\end{array}\right)^{\mathbf{T}}=\mathbf{A}_{l}\left(\begin{array}{ll}x_{1 g l} & y_{1 g l}\end{array}\right)^{\mathbf{T}}$
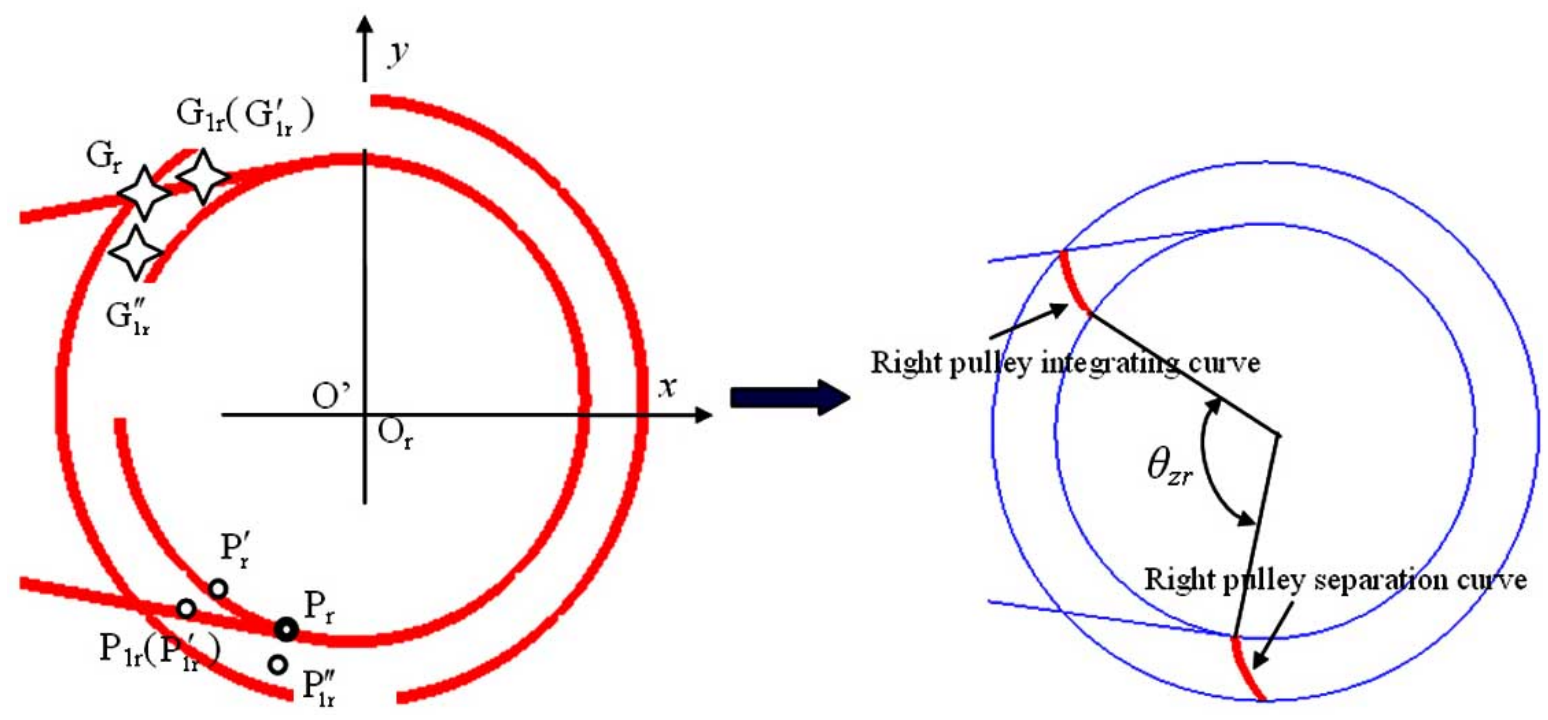

where, $x_{g l}$ and $y_{g l}$ are corresponding $x$-coordinate and $y$ coordinate of point $\mathrm{G}_{11}^{\prime \prime}$ in coordinate system $\mathrm{S}_{l}$.

\section{EQUATION OF THE RIGHT PULLEY CURVE}

\subsection{Separation Curve Equation}

The derivation of the right pulley separation curve equation is similar to that of the left one. As shown in Fig. (4), $\omega_{r}$ is the angular velocity of the right pulley and $t_{0 r}$ is the time the separation process spends, $\theta_{r}$ is the angle offset of coordinate system $\mathrm{S}_{r}$ during the separation process. So calculating relations about $\omega_{r}$ and $\theta_{r}$ are obtained in the following formulas.

$\omega_{r}=\frac{\sqrt{\left(r^{2}-r_{r}^{2}\right)}}{r_{r} t_{0 r}}$

$\theta_{r}=\omega_{r} t \frac{180}{\pi}$

Point $\mathrm{P}_{\mathrm{r}}\left(x_{0 p r}, y_{0 p r}\right)$ is the starting separating point of belt and pulley, $\mathrm{P}_{1 \mathrm{r}}\left(x_{1 p r}, y_{1 p r}\right)$ is any point before the end of the separation. Based on kinematics relationship, the speed of rolling pin can be described as $r_{r} \omega_{r}$. According to the geometric relationship, the coordinate of Point $\mathrm{P}_{1 \mathrm{r}}$ is described as:

$$
\begin{aligned}
& x_{1 p r}=x_{0 p r}-r_{r} \omega_{r} t \cos (\alpha) \\
& y_{1 p r}=y_{0 p r}+r_{r} \omega_{r} t \sin (\alpha)
\end{aligned}
$$

where, $x_{1 \mathrm{p} r}$ and $y_{1 \mathrm{pr}}$ are the corresponding $x$-coordinate and $y$ coordinate of point $\mathrm{P}_{1 \mathrm{r}}$ in coordinate system $S^{\prime}$.

As left pulley's derivation, defining the coordinate of point $\mathrm{P}_{1 \mathrm{r}}^{\prime \prime}$ as $\left(x_{p r}, y_{p r}\right)$ in coordinate $\mathrm{S}_{r}$ and the coordinate transformation matrix as $\mathrm{A}_{r}$, the coordinate $\left(x_{p r}, y_{p r}\right)$ is described as:

$$
\left(\begin{array}{ll}
x_{p r} & y_{p r}
\end{array}\right)^{\mathbf{T}}=\mathbf{A}_{\mathbf{r}}\left(\begin{array}{ll}
x_{1 p r} & y_{p 1 r}
\end{array}\right)^{\mathbf{T}}
$$

where, $\mathrm{A}_{r}$ is the coordinate transformation matrix from coordinate system $S^{\prime}$ to $\mathrm{S}_{l}$, the matrix is described as,

Fig. (4). Points relation diagram of right pullet. 
$A_{r}=\left[\begin{array}{cc}\cos \theta_{r} & -\sin \theta_{r} \\ \sin \theta_{r} & \cos \theta_{r}\end{array}\right]$

When describing relations in the fixed coordinate system $\mathrm{S}$, the offset from the right pulley coordinate origin to the left is $a$. So the coordinate equation is described as:

$$
\left(\begin{array}{ll}
x_{p r} & y_{p r}
\end{array}\right)^{T}=A_{r}\left(\begin{array}{ll}
x_{1 p r} & y_{1 p r}
\end{array}\right)^{T}+a\left(\begin{array}{ll}
1 & 0
\end{array}\right)^{T}
$$

\subsection{Integration Curve Equation}

Similar to the deduction of the left pulley, right pulley's rotation speed, time cost in the integration process and the angle right pulley rotating during integrating period, are the same as those of right pulley during separation process, whose parameters are $t_{0 \mathrm{r}}, \omega_{r}$ and $\theta_{r}$. As shown in Fig. (4), point $\mathrm{G}_{\mathrm{r}}\left(x_{0 \mathrm{gr}}, y_{0 \mathrm{gr}}\right)$ is the initial integration point between the left pulley and belt, point $\mathrm{G}_{1 \mathrm{r}}\left(x_{1 g r}, y_{1 g r}\right)$ is some point before the end of integration. According to the geometric relationship, the coordinate of point $\mathrm{G}_{1 \mathrm{r}}$ is described as:

$x_{1 g r}=x_{0 g r}+r_{r} \omega_{r} t \cos (\alpha)$

$y_{1 g r}=y_{0 g r}+r_{r} \omega_{r} t \sin (\alpha)$

where, $x_{1 p r}$ and $y_{1 p r}$ are the corresponding $x$-coordinate and $y$ coordinate of point $\mathrm{G}_{1 \mathrm{r}}$ in coordinate system $S^{\prime}$.

In a similar way, defining the coordinate of point $\mathrm{G}_{1 \mathrm{r}}^{\prime \prime}$ as $\left(x_{g r}, y_{g r}\right)$ in coordinate $\mathrm{S}_{r}$, the coordinate of point $\mathrm{G}_{1 \mathrm{r}}^{\prime \prime}$ is described as:

$\left(\begin{array}{ll}x_{g r} & y_{g r}\end{array}\right)^{\mathbf{T}}=\mathbf{A}_{\mathbf{r}}\left(\begin{array}{ll}x_{1 g r} & y_{1 g r}\end{array}\right)^{\mathbf{T}}$

When describing relations in the fixed coordinate system $\mathrm{S}$, the coordinate equation is described as:

$\left(\begin{array}{ll}x_{g r} & y_{g r}\end{array}\right)^{T}=A_{r}\left(\begin{array}{ll}x_{1 g r} & y_{1 g r}\end{array}\right)^{T}+a\left(\begin{array}{ll}1 & 0\end{array}\right)^{T}$

\section{MESHING CURVE AND ROLLING PIN NUMBER}

During driving, sliding shouldn't occur between pulleys and belt. In other words, rolling pin shouldn't slide on the pulley. So, it is deduced that initial separation point of the separation curve must superimposes with the starting point of the integration curve and the whole meshing curve is the combination of above two curves. Fig. (5) shows several

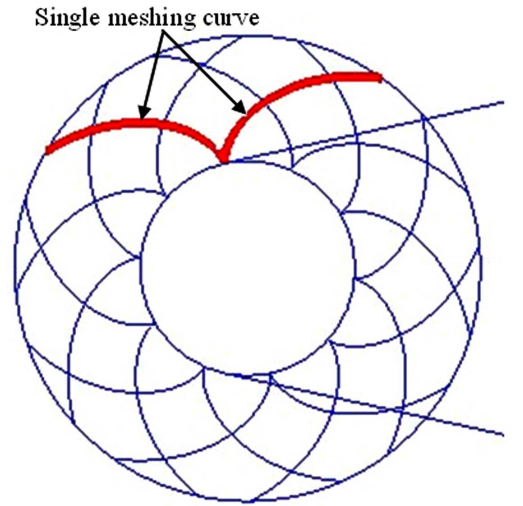

Fig. (5). Evenly placed meshing curves on the left pulley. meshing curves placed evenly on the left pulley and the thickened curve is one single meshing curve.

The initial separation point and the ending integration point of each pulley are in the same respective working cycle, and offset angles in their respective coordinates are expressed as $\theta_{z l}$ and $\theta_{z r}$, as shown in Fig. (2) and Fig. (3). With affine transformation, ending integration point could superimpose with initial separation point on each pulley by means of counterclockwise rotation. So there is the following relationship between $\left(x_{0 p l}, y_{0 p l}\right)$ and $\left(x_{1 g l}, y_{1 g l}\right)$

$\left(\begin{array}{ll}x_{0 p l} & y_{0 p l}\end{array}\right)^{\mathbf{T}}=A_{z l} \mathbf{A}_{l}\left(\begin{array}{ll}x_{1 g l} & y_{1 g l}\end{array}\right)^{\mathbf{T}} \mid t=t_{0 l}$

where, $\mathrm{A}_{z r}$ is affine transformation matrix of left pulley from the ending integration point to the initial separation point. It is described as:

$A_{z l}=\left[\begin{array}{cc}\cos \theta_{z l} & -\sin \theta_{z l} \\ \sin \theta_{z l} & \cos \theta_{z l}\end{array}\right]$

The relationship between $\left(x_{0 p r}, y_{0 p r}\right)$ and $\left(x_{1 g r}, y_{1 g r}\right)$ is similar.

$\left(\begin{array}{ll}x_{0 p r} & y_{0 p r}\end{array}\right)^{\mathbf{T}}=\left.A_{z r} \mathbf{A}_{l}\left(\begin{array}{ll}x_{1 g r} & y_{1 g r}\end{array}\right)^{\mathbf{T}}\right|^{t=t_{0 r}}$

where, $\mathrm{A}_{z r}$ is affine transformation matrix of right pulley from the ending integration point to the initial separation point. It is described as:

$A_{z r}=\left[\begin{array}{cc}\cos \theta_{z r} & -\sin \theta_{z r} \\ \sin \theta_{z r} & \cos \theta_{z r}\end{array}\right]$

The complete meshing equation is shown as follows:

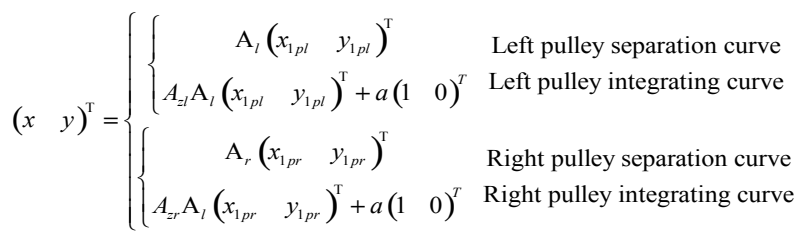

According to the geometric relationship, the belt length is calculated as:

$L=r_{l} \pi\left(1-\frac{\alpha}{90}\right)+2 a \cos (\alpha)+r_{r} \pi\left(1+\frac{\alpha}{90}\right)$

Suppose the number of rolling pins is $n$, and meshing curves are evenly distributed in the pulley, and the number of meshing curves is $n_{l}$ on the left pulley and $n_{r}$ on the right, the relations are as follows:

$$
\begin{aligned}
& l_{n}=\frac{L}{n} \\
& l_{n} n_{l}=2 \pi r_{l} \\
& l_{n} n_{r}=2 \pi r_{r}
\end{aligned}
$$

Then, the parameter $n$ can be solved and expressed as follows. 


$$
\begin{aligned}
n=\frac{r_{l} \pi\left(1-\frac{\alpha}{90}\right)+2 a \cos (\alpha)+r_{r} \pi\left(1+\frac{\alpha}{90}\right)}{2 \pi r_{l}} n_{l} \\
=\frac{\pi\left(1-\frac{\alpha}{90}\right)+2 \frac{a}{r_{l}} \cos (\alpha)+\frac{r_{r}}{r_{l}} \pi\left(1+\frac{\alpha}{90}\right)}{2 \pi} n_{l}
\end{aligned}
$$

By setting priorities and adjusting values of three parameter $r_{l}, r_{r}$ and $a$, a series of discrete solutions are calculated to meet integer parameters: $n, n_{l}$ and $n_{r}$.

\section{CONCLUSIONS}

Improving driving torque and reducing slip rate have important and practical significance in power transmission industries. This paper presents a new way that is evenly placed rolling pins on the belt and engraved curve trajectories on pulley. Firstly, separation and integration curves are deduced in the paper, and then, meshing curve equations are established. Finally, the method to determine the number of trajectories and rolling pins is obtained. All those can be used for mechanism design and further research.

\section{CONFLICT OF INTEREST}

None declared.

\section{ACKNOWLEDGEMENT}

None declared.

\section{REFERENCES}

[1] A.H. Mei, and J.H. Wang, "Research for the Dynamic designing of the Transmission of Synchronizer Gear-belt", J. Mach. Des. Res., vol. 15 , pp. $56-57,1999$.

[2] Z. Wang, "The calculation and the effect of elastic slide on efficiency in belt transmission", J. Mech. Transm., vol. 33, pp. 6364, 2009.

[3] G.L. Pu, and M.G. Ji, Mechanic Design. Beijing: Higher Education Press, China, 2006.

[4] Y. Zhang, "Dynamic research on serpentine belt drive systems", M.S. thesis, Northwestern Polytechnical University, XiAn, China, 2007.

[5] M. Jiang, "Capability research on automotive synchronous belt", M.S. thesis, Changchun University of Technology, ChangChun, China, 2009.

[6] P. Zhou. "Automotive synchronous belt drives design method and drives capability research", M.S. thesis, Changchun University of Technology, ChangChun, China, 2008.

[7] P.L. Cong. "Design of new ordinary v-belt CVT", M.S. thesis, Dalian Jiao Tong University, Dalian, China, 2008.

[8] Y.K. Wang, Z.J. Yang, L.N. Li, and X.C. Zhang, "The equation of meshing of spiral bevel gears manufactured by generating-line method", Open Mech. Eng. J., vol. 5, pp. 51-5, 2011. [Online] Available: http://www.benthamscience.com [Accessed Sept. 1, 2011].

[9] J.S. Zhao, Z.J. Feng, and F.L. Chu. Analytical theory of degree of freedom for robot mechanisms. Beijing: Science Press, China 2009.

[10] J. Oprea. Differential geometry and its applications. Beijing: Machinery Industry Press, China, 2006.

[11] Y.P. Cheng, K.Y. Zhang, and Z. Xu, Matrix Theory Xi. An: Northwestern Polytechnical University Press, China, 2006.

[12] C. Steger, M. Ulrich, and C. Wiedemann. Machine Vision Algorithms and Applications. Beijing: Tsinghua University Press, China, 2008.

Received: September 09, 2011

Revised: October 16, 2011

Accepted: November 18, 2011

(C) Zhao and Zhang; Licensee Bentham Open.

This is an open access article licensed under the terms of the Creative Commons Attribution Non-Commercial License (http://creativecommons.org/licenses/by$\mathrm{nc} / 3.0 /$ ), which permits unrestricted, non-commercial use, distribution and reproduction in any medium, provided the work is properly cited. 